\title{
España vs. Portugal en educación. Una aproximación sistémica
}

\author{
Francisco López Rupérez ${ }^{1}$ [D ; Isabel García García ${ }^{1}$ \\ ${ }^{1}$ Universidad Camilo José Cela (UCJC), España.
}

\begin{abstract}
Resumen. Como consecuencia de las exigencias del contexto y de su rápida transformación, las sociedades modernas se hallan confrontadas a la necesidad imperiosa de mejorar sus sistemas educativos. Una práctica de utilidad para la mejora de las organizaciones consiste en compararse con otras que, partiendo de situaciones inferiores, han conseguido avanzar de un modo sustantivo; para, posteriormente, tratar de averiguar cómo lo hicieron. El presente trabajo asume esa estrategia y adopta Portugal como elemento de comparación. Para ello, parte de una aproximación sistémica a la educación sobre la base de la estructura: contexto, inputs, políticas, outputs, y outcomes, que sirve como marco conceptual para seleccionar un conjunto amplio de indicadores sobre el que efectuar una comparación suficientemente sistemática entre los dos países, en materia educativa. Del análisis de las diferencias emergen las siguientes conclusiones: (a) en materia de variables de contexto, España se sitúa por encima de Portugal; (b) en materia de inputs, Portugal supera a España en gasto acumulado por alumno y está retrasada en dotación de recursos educativos y humanos; (c) en cuanto a outputs y a outcomes, Portugal supera a España en todos los indicadores considerados; (d) finalmente, en relación con las políticas, la posición de Portugal está claramente más adelantada que la de España. Estas evidencias se traducen en un conjunto de recomendaciones, inspiradas en el consenso internacional y validadas por el éxito del caso portugués.
\end{abstract}

Palabras clave: educación comparada; enfoque sistémico; educación española; educación portuguesa; evaluación.

Espanha vs. Portugal na educação. Uma abordagem sistêmica

Resumo. Como consequência das exigências do contexto e de sua rápida transformação, as sociedades modernas são confrontadas com a necessidade urgente de melhorar seus sistemas educacionais. Uma prática útil para o aprimoramento das organizações consiste em comparar-se com outras que, partindo de situações inferiores, conseguiram avançar de forma substancial; para, depois, tentar descobrir como o fizeram. O presente trabalho assume essa estratégia e adota Portugal como elemento de comparação. Para isso, parte de uma abordagem sistêmica da educação baseada na estrutura: contexto, inputs, políticas, outputs e outcomes, que serve como uma estrutura conceitual para selecionar um amplo conjunto de indicadores sobre o qual efetuar uma comparação suficientemente sistemática entre os dois países, em matéria de educação. Da análise das diferenças, emergem as seguintes conclusões: (a) em termos de variáveis de contexto, a Espanha está acima de Portugal; (b) em termos de inputs, Portugal supera a Espanha na despesa acumulada por aluno e fica atrás na oferta de recursos educativos e humanos; (c) em relação a outputs e a outcomes, Portugal supera a Espanha em todos os indicadores considerados; (d) finalmente, em relação às políticas, a posição de Portugal está claramente à frente da Espanha. Estas evidências traduzem-se num conjunto de recomendações, inspiradas em consensos internacionais e validadas pelo sucesso do caso português.

Palavras-chave: educação comparada; abordagem sistêmica; educação espanhola; educação portuguesa; avaliação.

Spain vs. Portugal in education. A systemic approach

Abstract. As a result of the demands of the context and its rapid transformation, modern societies have been confronted with the urgent need to improve their educational systems. A useful practice for the improvement of organizations is to compare oneself with others that, starting from inferior situations, have managed to advance in a substantive way; to later try to find out how they did it. This paper takes up this strategy and adopts Portugal as an element of comparison. To do this, it starts from a systemic approach to education based on the structure: context, inputs, policies, outputs, and outcomes, which serves as a conceptual framework for selecting a broad set of indicators on which to make a sufficiently systematic comparison between the two countries, in in the field of education. The following conclusions emerge from the analysis of the differences: (a) regarding context variables, Spain is above Portugal; (b)in terms of inputs, Portugal exceeds Spain in accumulated expenditure per student and is behind in providing educational and human resources; (c) in terms of outputs and outcomes, Portugal exceeds Spain in all the indicators considered; (d) 
finally, in relation to policies, the position of Portugal is clearly more advanced than that of Spain. This evidence is translated into a handful of recommendations, inspired by international consensus and validated by the success of the Portuguese case.

Keywords: comparative education; systemic approach; spanish education; portuguese education. evaluation.

\section{Introducción}

En el contexto de la llamada "cuarta revolución industrial" (Shwab, 2016) -una revolución tecnológica basada en sistemas físicos, sistemas biológicos, sistemas digitales y en sus interacciones- las sociedades modernas se hallan confrontadas a la necesidad imperiosa de mejorar sus sistemas educativos (Schleicher, 2018). Este consenso internacional emergente se ha visto reforzado por los efectos deletéreos de la pandemia COVID-19 (Reimers y Schleicher, 2020).

Aun cuando una práctica habitual en la gestión para la mejora de las organizaciones consiste en compararse con aquellas otras situadas en posiciones superiores (López Rupérez, 1994), es posible, asimismo, modificar la orientación y compararse con las que, partiendo de situaciones inferiores, han conseguido avanzar de un modo sustantivo, para, posteriormente, tratar de averiguar cómo lo hicieron.

Geográfica, cultural y lingüísticamente próximo, Portugal es un país hermano de un tamaño intermedio que ha padecido como España los efectos de una dictadura militar; ha arrastrado un retraso histórico superior; dispone de un inferior nivel de riqueza medida por el PIB per cápita, y un índice de nivel socioeconómico y cultural más bajo. Y, a pesar de todas esas desventajas comparativas, Portugal ha conseguido evolucionar a mejor a lo largo de las últimas décadas y obtiene resultados superiores a los de España en materia educativa, medidos por diferentes indicadores que son considerados clave en el panorama internacional.

Por tal motivo, procede efectuar una comparación sistemática entre los dos países en el ámbito de la educación escolar, intentar evidenciar algunas diferencias y apuntar a sus muy probables factores explicativos. Además, y bajo este objetivo, en el presente trabajo late la pretensión de arrojar alguna luz sobre la naturaleza de las reformas que debería emprender España en la próxima década en materia educativa y sus rasgos ineludibles, tomando como inspiración el caso portugués. 
De acuerdo con este propósito, la metodología de análisis de este trabajo se apoya en una aproximación sistémica a la educación que servirá como marco conceptual para seleccionar un conjunto relativamente amplio de indicadores sobre el que efectuar una comparación sistemática entre los dos países, en materia educativa. Esta comparación reposará, principalmente, en la consideración de series cronológicas. De los resultados obtenidos y del estudio de las diferencias emerge un conjunto de conclusiones que se traducirá en un puñado de recomendaciones inspiradas en el consenso internacional, y validadas por el éxito del caso portugués.

\section{Una aproximación sistémica a la Educación}

La aproximación sistémica a la Educación es deudora del enfoque adoptado por el mundo de los indicadores internacionales de educación que sirvió como elemento estructurante del proyecto INES (Indicators of Education Systems) de la OCDE (CERI-OECD,1992;1994). La publicación anual Education at a Glance. OECD Indicators se articuló, en buena parte de sus ediciones, sobre la base de dicha aproximación sistémica (OECD, 2012).

En el presente trabajo, partiremos de la configuración básica de ese enfoque sistémico y lo ampliaremos, profundizando en sus relaciones internas y aproximándonos a su nivel de complejidad.

El sistema educativo propiamente dicho puede modelizarse como un conjunto de inputs, entrantes o insumos que, mediante los procesos y las políticas que se desarrollan en su seno, generan un conjunto de outputs o resultados propiamente educativos. Este esquema simple se complica (véase la figura 1) al considerar que no sólo la naturaleza y magnitud de los inputs permiten el desarrollo de los procesos y las políticas y, con toda probabilidad, los condicionan, sino que, inversamente, las políticas operan sobre los inputs en tanto que actuaciones de los gobiernos que los proporcionan y administran. En un sentido análogo, la aplicación de políticas sobre el sistema producirá resultados educativos; pero, en cualquier sistema moderno e inteligente, los resultados actuarán retroactivamente sobre las políticas -a partir de los mecanismos de feedback formativo- permitiendo reforzarlas o corregirlas (López Rupérez et al., 2019).

Esta complejidad se incrementa cuando se consideran dos elementos externos al sistema propiamente dicho, pero en interacción con él y entre sí. Por un lado, está el entorno o contexto socioeconómico y cultural en el que opera el sistema educativo y que alude a un sistema más amplio; y, por otro, los outcomes, consecuencias o impacto que tiene la educación 
más allá del sistema educativo. El contexto condiciona la magnitud de los inputs, incide sobre las políticas y afecta a los resultados, por una vía tanto directa -por efecto de factores diversos propios de ese sistema más amplio del que la educación es tan solo un subsistema-, como indirecta, mediante su incidencia sobre la definición e implementación de las políticas educativas que operan sobre los resultados.

Por otra parte, las consecuencias de los outputs del sistema educativo derivan obviamente -al menos en parte- de dichos resultados, aunque esta vinculación entre resultados y consecuencias esté modulada por la influencia del contexto. Pero, además, esos cambios producidos en variables sociales y económicas reflejan, a la postre, cambios del contexto; de modo que la educación influye sobre el contexto y es influido por él, de acuerdo con un bucle causal que se extiende en el medio y largo plazo, y que definirá un horizonte de progreso si el sistema tiene éxito.

Figura 1. Modelo conceptual sistémico de la Educación ampliado.

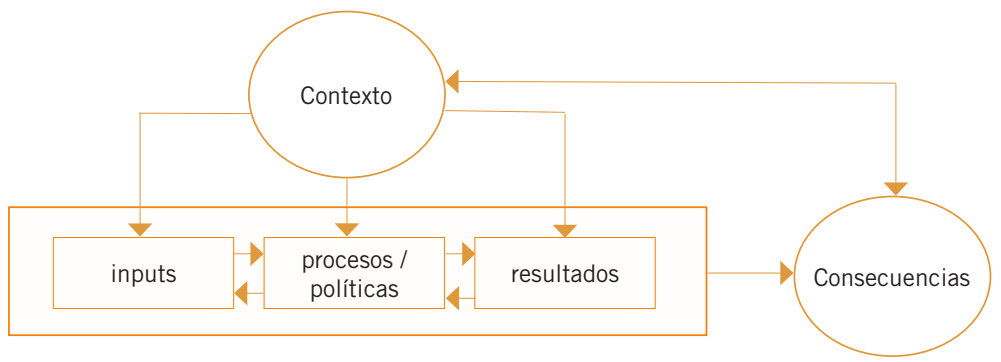

Fuente: Elaboración propia (López Rupérez y García García, 2020).

Disponemos, por tanto, de un modelo conceptual integrado, como el que se representa en la figura 1, que nos servirá de referente para articular el presente estudio comparado.

\section{Una comparación sistemática entre España y Portugal en materia educativa}

Por su potencia, a la hora de realizar una descripción completa de los sistemas educativos, el anterior modelo conceptual resulta especialmente apropiado para una comparación sistemática entre España y Portugal en materia educativa. En lo que sigue, efectuaremos la comparación sobre la base de esos cinco elementos que aparecen representados en la figura 1: Contexto, inputs, outputs, outcomes y políticas. 


\subsection{Desde la perspectiva del contexto}

\subsubsection{Nivel socioeconómico y cultural}

Una de las variables contextuales ampliamente reconocidas, por su impacto sobre los resultados de los alumnos, es el nivel socioeconómico y cultural. Desde su primera edición, PISA ha tomado en consideración el índice correspondiente (ESCS: Economic, Social and Cultural Status) y lo ha medido de un modo sistemático a partir del cuestionario dirigido a los alumnos (OECD, 2019). La tabla 1 -o cuadro de yuxtaposición- muestra los resultados comparados España/Portugal en relación con dicho índice (ISEC en español). EI ISEC (Índice Socio Económico y Cultural) es un indicador, normalizado tomando como base los países miembros de la OCDE, con un valor medio igual a 0 y una desviación estándar igual a 1 , de modo que valores positivos de dicho índice significan que el país se sitúa por encima de la media, y valores negativos significan que se sitúa por debajo de ella.

\subsubsection{Nivel de riqueza}

El nivel de riqueza de un país es otra de las variables contextuales clásicas que se mide habitualmente mediante su PIB per cápita. Al tratarse de un indicador que toma en consideración la población, resulta mucho más preciso, a efectos de comparación, que el PIB total. La tabla 1 recoge los resultados para España y Portugal de dicho indicador de nivel de riqueza.

Con relación tanto a un indicador contextual, como al otro España se encuentra, con respecto a Portugal, en una posición más favorable, lo que refleja un mayor nivel de desarrollo.

\subsection{Desde la perspectiva de los inputs}

\subsubsection{Gasto acumulado por alumno}

El gasto acumulado por alumno en establecimientos escolares en las edades comprendidas entre los 6 y los 15 años es uno de los indicadores empleados por PISA para cuantificar el nivel de gasto educativo en términos comparables, cuando se corrige del efecto de las diferencias entre países en cuanto a sus respectivos poderes adquisitivos (paridad de poder adquisitivo, ppa). En la tabla 1 se muestran los valores correspondientes al ejercicio de 2015, que es el último consolidado y disponible para la comparación internacional (OCDE, 2019). Se advierte en ella que, a pesar de su inferior nivel de riqueza, Portugal gasta bastante más por alumno que España. 


\subsubsection{Dotación en personal educativo}

A partir de las respuestas al cuestionario dirigido a directores escolares, la edición 2018 de PISA ha introducido un "índice de escasez de personal educativo" (OECD, 2019) -también normalizado sobre la base de los países miembros de la OCDE- con un valor medio igual a 0 y una desviación estándar igual a 1. La tabla 1 recoge los valores correspondientes a este indicador para España y para Portugal.

\subsubsection{Dotación en recursos educativos}

Un tratamiento similar al anterior se ha efectuado en PISA 2018 para los recursos educativos (OECD, 2019) obteniéndose para España y para Portugal los valores que se muestran en la tabla 1 . Tanto para el indicador de dotación de personal, como para el de recursos educativos, los datos revelan índices de escasez superiores a la media de la OCDE. En ambos casos, el grado de escasez es mayor en Portugal que en España, según la percepción de los directores de los centros de educación secundaria encuestados.

Tabla 1. Indicadores de contexto y de inputs considerados en el estudio

\begin{tabular}{lccc}
\hline \multicolumn{1}{c}{ Indicador } & España & Portugal & OCDE \\
\hline - Nivel socioeconómico y cultural (ISEC) & $-0,1$ & $-0,4$ & 0,0 \\
- Nivel de riqueza (PIB pc en \$ USA ppa) & 33.629 & 28.760 & 39.333 \\
- Gasto acumulado/alumno (\$ USA ppa) & 74.947 & 83.050 & 90.294 \\
- Escasez en personal educativo & 0,33 & 0,82 & 0,0 \\
- Escasez en recursos educativos & 0,22 & 0,48 & 0,0
\end{tabular}

Nota: Los datos de nivel de riqueza y de gasto acumulado por alumnos (de 6 a 15 años) corresponden al año 2015 que es el último reportado por la OCDE.

Fuente: Elaboración propia sobre la base de datos recopilados por la OCDE (OECD, 2016; 2019)

\subsection{Desde la perspectiva de los outputs}

En un sentido restringido - pero más fácil de medir- los outputs de un sistema educativo son sus logros académicos, que pueden ser considerados según diferentes perspectivas: se pueden abordar desde un enfoque más global o más analítico; basados en pruebas de rendimiento o en estadísticas administrativas; deteniéndonos en la educación o pensando en sus consecuencias; mirando al presente o preparando el futuro; analizando la evolución del propio sistema o comparándonos con otros. Todas estas perspectivas, no excluyentes entre sí, serán consideradas en lo que sigue en nuestro particular ejercicio de comparación internacional. 
Por otro lado, es sabido que el impacto de las políticas educativas sobre los resultados del sistema, en su conjunto, se deja sentir en el medio y largo plazo; de ahí que, a efectos de comparación -sea de un país consigo mismo, sea con los demás-, resulte más seguro analizar series históricas, cuando menos a lo largo de una década. Este es el enfoque adoptado en el presente estudio para abordar la comparación España con Portugal en lo concerniente a los outputs de sus respectivos sistemas educativos.

\subsubsection{Rendimiento en PISA}

La tabla 2 -o cuadro de yuxtaposición- resume la comparación entre España y Portugal que permite la síntesis diagnóstica efectuada por PISA sobre la base de su historial -de cerca de dos décadas- relativo a los resultados de sus evaluaciones a gran escala (OECD, 2019). Del análisis de los datos cabe inferir las siguientes evidencias:

Tabla 2. Resumen comparado de las tendencias de rendimiento en PISA en España y en Portugal

\begin{tabular}{|c|c|c|c|c|c|c|}
\hline \multirow{2}{*}{\begin{tabular}{l}
\multicolumn{1}{c}{ Tendencias } \\
Trayectoria de rendi- \\
miento general
\end{tabular}} & \multicolumn{2}{|c|}{$\begin{array}{c}\text { Lectura } \\
\text { España Portugal }\end{array}$} & \multicolumn{2}{|c|}{$\begin{array}{c}\text { Matemáticas } \\
\text { España Portugal }\end{array}$} & \multicolumn{2}{|c|}{$\begin{array}{c}\text { Ciencias } \\
\text { España Portugal }\end{array}$} \\
\hline & - & $\begin{array}{l}\text { Constan- } \\
\text { temente }\end{array}$ & Plana & $\begin{array}{l}\text { Positiva, } \\
\text { pero } \\
\text { menos } \\
\text { en los } \\
\text { últimos } \\
\text { años }\end{array}$ & $\begin{array}{l}\text { Más } \\
\text { negativa } \\
\text { en los } \\
\text { últimos } \\
\text { años }\end{array}$ & $\begin{array}{l}\text { Positiva, } \\
\text { pero } \\
\text { menos } \\
\text { en los } \\
\text { últimos } \\
\text { años }\end{array}$ \\
\hline $\begin{array}{l}\text { Tendencia promedio } \\
\text { entre los estudiantes } \\
\text { con mejor rendimien- } \\
\text { to (percentil 90) }\end{array}$ & 1,2 & 4,1 & $-0,1$ & 7,8 & $-0,2$ & 6,0 \\
\hline $\begin{array}{l}\text { Tendencia promedio } \\
\text { entre los estudiantes } \\
\text { de bajo rendimiento } \\
\text { (percentil 10) }\end{array}$ & $-3,2$ & 5,2 & 0,3 & 2,6 & 0,4 & 1,7 \\
\hline $\begin{array}{l}\text { Brecha en los resul- } \\
\text { tados de aprendizaje } \\
\text { entre los estudiantes } \\
\text { con mayor y menor } \\
\text { rendimiento }\end{array}$ & - & Estable & Estable & $\begin{array}{l}\text { Brecha } \\
\text { cada vez } \\
\text { mayor }\end{array}$ & Estable & Estable \\
\hline
\end{tabular}

Fuente: elaboración propia a partir de PISA 2018 Results (Volume I): What Students Know and Can Do» (C) OECD 2019, Anexo D, p. 327.

a) España presenta una tendencia plana o ligeramente negativa en el periodo considerado, mientras que Portugal se ha comportado de forma consistentemente positiva en las tres áreas consideradas: Lectura, Matemáticas y Ciencias. 
b) En materia de excelencia (percentil 90), España está estancada mientras que Portugal ha mejorado significativamente en las tres áreas, particularmente en Matemáticas y en Ciencias.

c) En materia de equidad (percentil 10), España apenas si ha variado en Matemáticas y en Ciencias y ha empeorado en Lectura, mientras que Portugal ha mejorado en las tres áreas.

d) El comportamiento en Matemáticas se traduce en un cierto empeoramiento de Portugal en cuanto a la brecha existente entre los extremos, toda vez que la excelencia ha progresado más rápidamente que la equidad, lo que no sucede en España debido al carácter plano de su evolución.

\subsubsection{Tasa de la población joven que ha completado la educación secundaria superior}

La tasa de la población adulta joven (25-34 años) que ha completado, al menos, la educación secundaria superior es un indicador internacional de resultados que apunta al grado de acierto de un sistema educativo en la preparación del futuro, al referirse a un nivel formativo considerado imprescindible. La figura 2 fija la atención en la comparación de tendencias entre España y Portugal, tomando en ambos casos como referencia el comportamiento de la Unión Europea en su conjunto.

Figura 2. Evolución del porcentaje de jóvenes de 25 a 34 años que han completado al menos, el nivel educativo de la educación secundaria superior (CINE 3-8) en España, Portugal y Unión Europea. Años 2010 a 2019

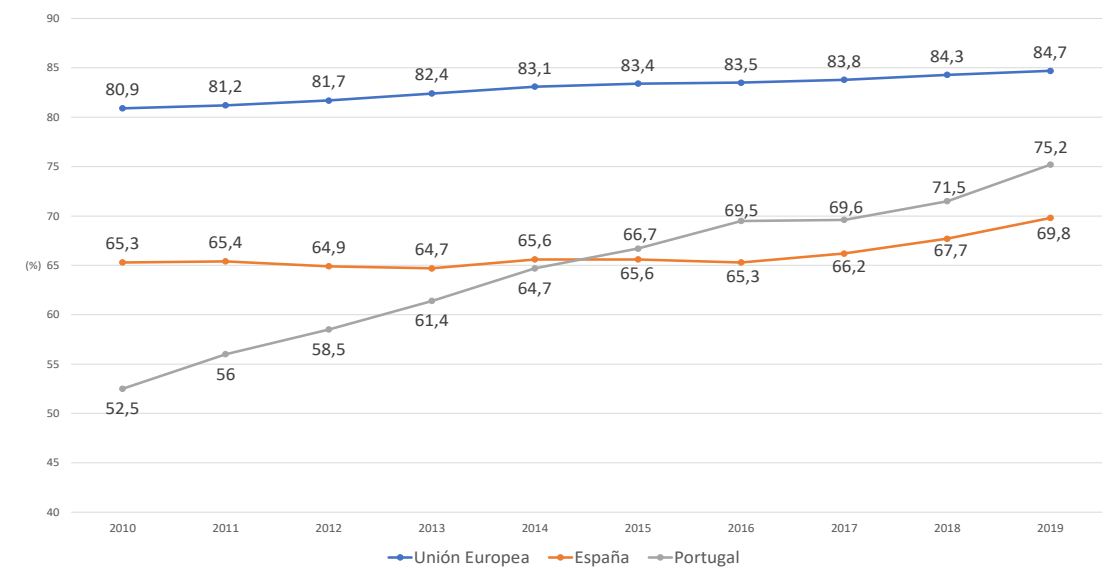

Fuente: Elaboración propia a partir de Eurostat [edat_Ifse_03]. 
Del análisis de los datos que se representan en dicha figura 2 cabe destacar las siguientes evidencias:

a) Portugal, que partía en 2010 de una posición retrasada 12,8 puntos porcentuales con respecto a España, le superaba en 5,2 puntos porcentuales en 2019.

b) Portugal, en este mismo periodo, ha reducido la brecha de los 28,4 puntos porcentuales que le separaban de la UE, a 10,1 puntos, habiendo logrado, por tanto, una convergencia de 18,3 puntos de magnitud. Mientras, España lo hacía de 15,6 a 15,5 puntos porcentuales, es decir, con una convergencia de 0,1 puntos, que no es significativa.

\subsubsection{Brecha formativa de la población joven}

Las figuras 3 y 4 muestran la evolución de los respectivos patrones de distribución de la población adulta joven (25-34 años) por niveles de formación, correspondientes a España y a Portugal. De su análisis cabe destacar los siguientes hechos:

a) España ha corregido en parte esa brecha, pero a un ritmo lento e incapaz de alterar en una década el patrón de distribución -en forma de $\mathrm{V}$ - de la población joven por niveles de formación: básico, medio y superior.

b) Portugal ha hecho avances mayúsculos en el mismo periodo de tiempo, reduciendo notablemente el porcentaje de la población joven con estudios básicos, y elevando a la par los porcentajes correspondientes a estudios medios y a estudios superiores. Esa mayor rapidez en los procesos de mejora se evidencia por la simple comparación gráfica de las anchuras de las correspondientes bandas de variación.

c) Los progresos efectuados por Portugal, a lo largo del periodo de referencia, en materia de tasa de la población adulta joven (25-34 años) que ha conseguido completar, al menos, los estudios correspondientes a la educación secundaria superior, se ha traducido en la corrección del patrón de distribución de esa misma población por niveles de formación, que al principio de dicho periodo se parecía al de España y ahora se parece mucho más al de la Unión Europea, con forma de V invertida.

d) El notable incremento producido en Portugal, en cuanto a porcentaje de población joven con un nivel intermedio de formación, le ha Ilevado a alcanzar los 37,6 puntos porcentuales frente a 23,2 de España. 
Figuras 3 y 4. Evolución del nivel de educación de la población comprendida en el intervalo de edad de 25 a 34 años en España y en Portugal, respectivamente. Años 2010-2019

España

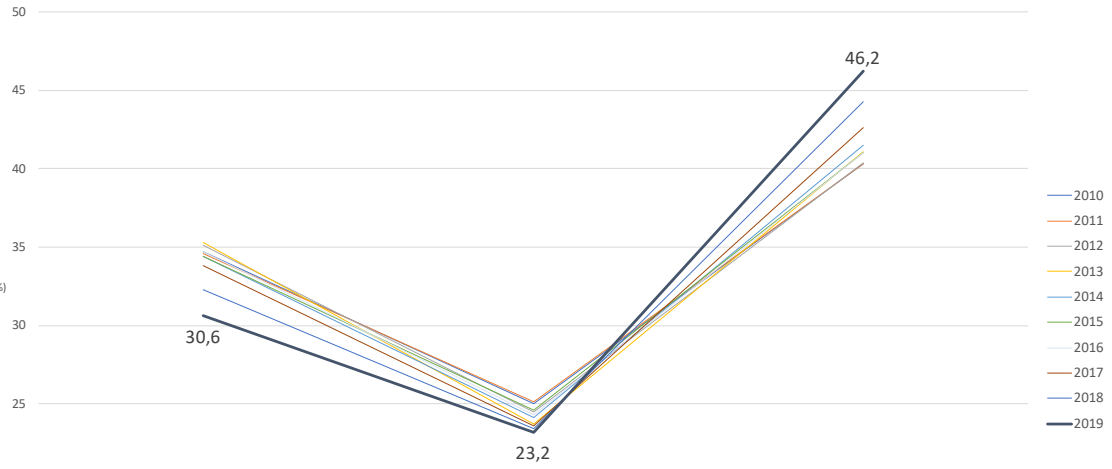

20

15

50

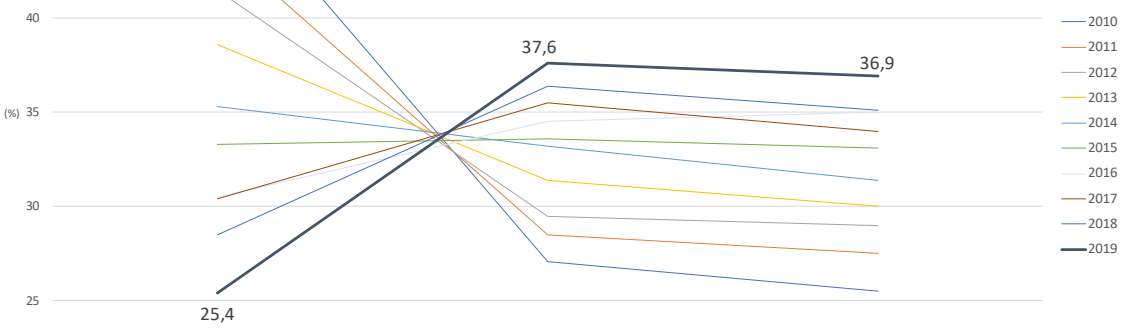

Fuente: Elaboración propia a partir de Eurostat [edat_Ifse_03].

\subsection{Desde la perspectiva de los outcomes}

De conformidad con la orientación típica de los sistemas de indicadores internacionales de educación (CERI-OCDE, 1992; 1994), los outcomes de un sistema educativo equivalen al impacto de dicho sistema que se proyecta en los ámbitos social y económico (CERI-OCDE, 2007), más allá de su marco concreto de definición. Aun cuando la relación entre outputs y outcomes está 
modulada por un conjunto diverso de factores intervinientes, de conformidad con la evidencia empírica disponible la intensidad de la asociación entre ambos es las más de las veces, aunque moderada, estadísticamente significativa (Xia y Kirby, 2009). Por tal motivo, procede considerar los outcomes como un producto, siquiera sea parcial, de los sistemas educativos.

\subsubsection{Abandono educativo temprano}

Este indicador, que forma parte destacada del Monitor de Educación y Formación -herramienta empleada por la Comisión Europea para el seguimiento de los países miembros en materia educativa (European Commission, 2019)-, apunta directamente, por su propia definición, al ámbito de los outcomes. De hecho, antes de que pasara a formar parte del conjunto de indicadores para el seguimiento de la Estrategia de Lisboa en materia educativa, el abandono educativo temprano era un indicador europeo de cohesión social (SC051).

En materia de abandono educativo temprano, la tendencia manifestada en los indicadores de outputs se hace, si cabe, aún más clara. La conclusión que se deriva de un modo evidente de los datos representados en la figura 5 es la siguiente: partiendo en 2009 de valores similares a los de España, Portugal ha logrado en el periodo de referencia converger prácticamente con la UE, mientras que España mantiene todavía una brecha de convergencia con la Unión de 6,9 puntos porcentuales y tendrá dificultades para alcanzar en 2020 el objetivo adaptado del 15 por ciento.

Figura 5. Evolución del abandono educativo en España, Portugal y Unión Europea. Años 2010 a 2019.

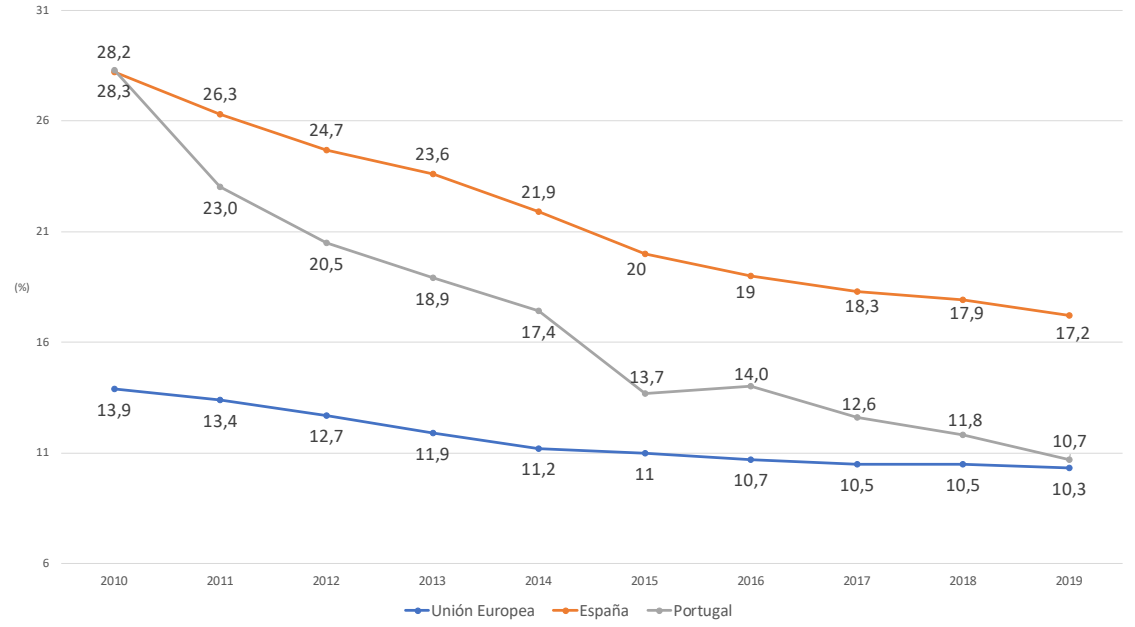

Fuente: Elaboración propia a partir de Eurostat [SDG_04_10]. 


\subsubsection{Tasa de NiNis}

Uno de los indicadores de la Unión Europea que integra, a la vez, situaciones de no-formación y de no-empleo en los jóvenes es el porcentaje de las personas de entre 18 y 24 años que ni estudia ni trabaja. Conocidos como NiNis, estos jóvenes son firmes candidatos a quedarse al margen del camino en su trayectoria vital lo que, al integrar sus efectos a nivel global, se convierte en una seria amenaza, tanto para la cohesión social como para el desarrollo social.

Figura 6. Evolución del porcentaje de la población joven con edades comprendidas entre 15 y 24 años que ni estudia ni trabaja en la Unión Europea, en España y en Portugal. Años 2010-2018

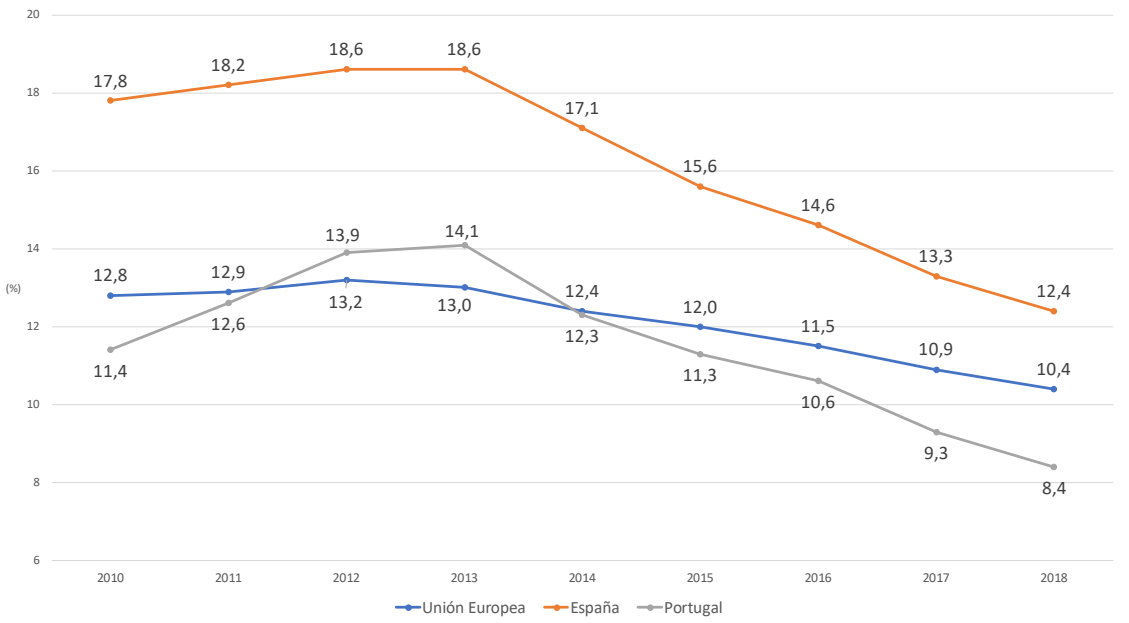

Fuente: Elaboración propia a partir de Eurostat [lfsi_neet_a].

El análisis de los datos correspondiente a la evolución de la tasa de NiNis para España y Portugal -y para la UE como referencia de fondo- se representan gráficamente en la figura 6. De la consideración de esas tres series cronológicas se infieren las siguientes evidencias:

a) España se sitúa, en cualquier punto de la serie, sistemáticamente por encima de Portugal como consecuencia, muy probablemente, de la desventaja de España en materia tanto de abandono educativo temprano como de desempleo juvenil, así como debido a su interacción, por efecto de esa regla empírica que establece "a menor formación, mayor desempleo". 
b) En el periodo central de la crisis de 2008 el ritmo de crecimiento de la tasa de NiNis fue muy superior en Portugal que en España; pero a la salida de aquélla Portugal había sido capaz de mejorar notablemente su situación hasta colocarse, frente al fenómeno $\mathrm{NiNi}$, en una posición más ventajosa que la de la propia UE.

\subsubsection{Riesgo de pobreza o exclusión social}

De conformidad con la Estrategia Europa 2020, se consideran personas en riesgo de pobreza y/o exclusión social a la población que se encuentra en cualquiera de las situaciones siguientes: (a) Personas que viven con bajos ingresos ( $60 \%$ de la mediana del ingreso equivalente o por unidad de consumo en el año anterior a la entrevista); (b) personas que sufren privación material severa (4 de los 9 ítems definidos); (c) personas que viven en hogares con una intensidad de empleo muy baja (por debajo del $20 \%$ del total de su potencial de trabajo en el año anterior a la entrevista). En el caso de que las personas estén incluidas en más de una de estas tres condiciones, se contabilizan solo una vez (Eurostat, 2020).

El análisis de la evolución de este fenómeno por niveles de formación puede arrojar alguna luz sobre el comportamiento diferenciado entre España y Portugal a este respecto y sobre algunas de sus claves explicativas. Las figuras 7 y 8 permiten esa comparación más compleja. Un resultado surge con nitidez de su análisis y es que, aun cuando el riesgo de pobreza o exclusión social de la población joven (18-24 años) afecta en mayor medida a España, sea cual fuere el nivel de formación considerado, la diferencia más clara, tanto en magnitud como en tendencia, corresponde al sector de más bajo nivel de formación. Así, se advierte con toda claridad que conforme en Portugal la tasa correspondiente empezó a reducirse desde un valor de 51,9 puntos porcentuales a partir de 2013, en España sucedió dos años después y desde un valor de 60,9 puntos.

A la vista de lo anterior, parece como si, en comparación, el riesgo de pobreza o exclusión social se cebara especialmente en España con los sectores de más bajo nivel de formación; lo que, junto con el poder predictivo del abandono educativo temprano en relación con dicho indicador (López Rupérez y García García, 2020) parece sugerir -con todas las limitaciones que para este tipo de evidencias plantea la atribución causal- el promover, con cierta urgencia, un incremento del nivel de formación de esos jóvenes situados en el nivel inferior y una mejora de sus cualificaciones. 
Figuras 7 y 8. Evolución del riesgo de pobreza o exclusión social de la población joven con edades comprendidas entre 18 y 24 años, según el nivel educativo, en España y Portugal, respectivamente . Años 2008-2018

\section{España}
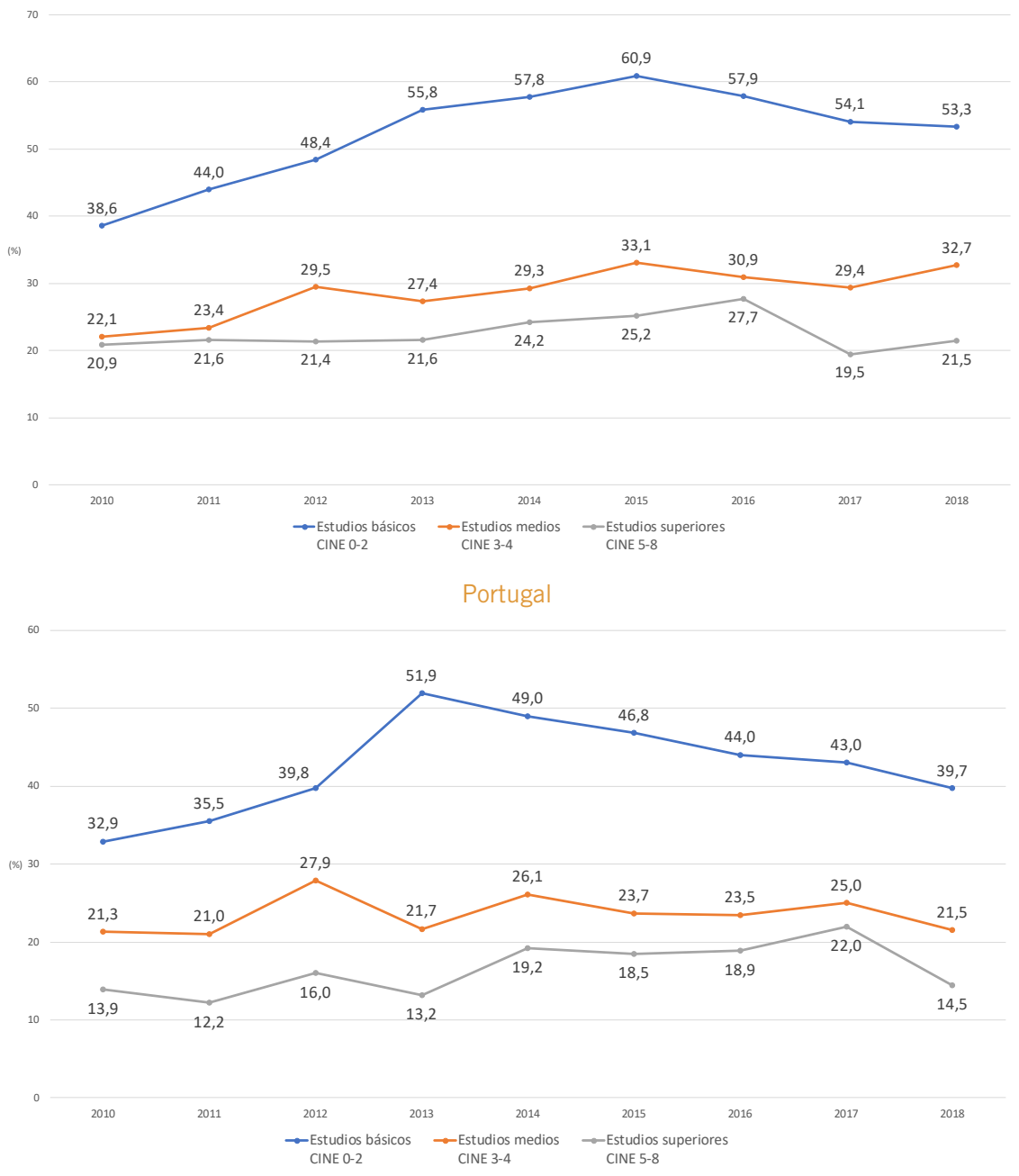

Fuente: Elaboración propia a partir de Eurostat [ilc_peps04].

\subsection{Desde la perspectiva de las políticas}

Aun cuando la influencia de las políticas sobre los resultados de los alumnos está mediada por un conjunto amplio de niveles y de factores intermedios, su intensidad -o fuerza de la relación entre políticas y outputs- ha de considerarse complementada por su extensión, que se refiere a su alcance, 
medido por la amplitud de la población a la que llega dicha influencia. Así que la relevancia de un factor, o de una política educativa que lo potencie, dependerá del producto intensidad x extensión (López Rupérez, 2020).

Por tal motivo, procede profundizar en los análisis de las políticas porque, con una muy elevada probabilidad, se encuentren en ellas algunas de las claves de las diferencias antes descritas en materia de resultados.

\subsubsection{Algunas características de las reformas educativas portuguesas}

Un análisis del caso portugués, apoyado en distintas fuentes (Crato, 2020; OECD, 2018), permite concluir que los sucesivos gobiernos, de signos políticos diferentes, han prestado a la educación un interés especial, y han introducido reformas bien alineadas con objetivos de mejora situados en el medio y largo plazo; mejoras que se han puesto de manifiesto, con bastante claridad, en el anterior análisis empírico de las series cronológicas.

Una de las reformas extensas que el Gobierno portugués emprendió al final de la primera década del presente siglo, y que en este punto conviene traer a colación, fue la extensión de la enseñanza obligatoria hasta los 18 años. El 27 de agosto de 2009, la Asamblea de la República aprobó la Ley $n^{\circ} 85 / 2009$ que amplió la obligatoriedad y gratuidad de la enseñanza a un periodo de 12 años (6-18 años). Con ello, se modernizaba la reforma que en 1986 extendió la etapa obligatoria hasta los 16 años pero que tardó un decenio en ser implantada. Por su carácter masivo, por su impulso ecualizador de los territorios y por su naturaleza de política de Estado, es altamente probable que algunos de los resultados observados en cuanto al incremento del nivel formativo de los jóvenes -particularmente la reducción del abandono educativo temprano y la reducción de la brecha formativa de la población joven- estén vinculados a esa valiente decisión política, que hubo de implementarse en un contexto de reducción drástica del gasto público por efecto de la intervención de la economía portuguesa derivada de la gran crisis de 2008. Con esta reforma -alineada con una recomendación genérica del Parlamento europeo del 1 de diciembre de 2011 (Parlamento Europeo, 2011)-, Portugal se incorporaba al grupo relativamente numeroso de países y de regiones de la Unión Europea que han adoptado una decisión semejante (Eurydice, 2015).

Esta iniciativa se suma a un conjunto amplio de reformas continuadas, acumulativas y coherentes, iniciadas con el comienzo del siglo XXI, cuyos principios mayores y sus concreciones pueden sintetizarse -a partir del análisis histórico efectuado por Crato (2020)- en los términos siguientes: 
a) Prestar una atención prioritaria a los resultados.

- Difusión pública de los resultados de cada centro en las pruebas nacionales de final de la educación secundaria.

- Atención especial a los resultados en la orientación de los aprendizajes básicos.

- Definición de un sistema de estándares que especifiquen lo que los alumnos tienen que alcanzar.

- Establecimiento de un sistema integrado de evaluaciones externas e internas para la mejora.

- Fortalecimiento de la autorregulación del sistema mediante las evaluaciones externas.

- Creación de un Instituto de Evaluación Educativa como organismo autónomo e independiente.

b) Conciliar calidad con cantidad.

- Extensión de la enseñanza obligatoria hasta los 18 años.

- Extensión de la enseñanza del inglés.

- Incremento del gasto educativo hasta el 5,1\% de su PIB, recuperando el nivel anterior a la crisis.

c) Conciliar excelencia con equidad.

- Asunción colectiva de la idea de que la escuela puede hacerlo mejor.

- Definición de un currículo bien estructurado y exigente, y el impulso de una educación ambiciosa para todos.

- Desarrollo de un plan para reducir el abandono educativo y apoyar a los alumnos con dificultades académicas y de entornos desaventajados.

- Apoyo en una educación seria y una evaluación fiable, de acuerdo con estándares nacionales.

- Implementación de una concepción flexible de la formación profesional, asociada a las empresas.

d) Hacer del conocimiento lo primero.

- Considerando que el conocimiento es importante.

- Asumiendo que, sin una base robusta de conocimiento, los alumnos no podrán desarrollar competencias avanzadas. 
- Reconociendo que la amplitud de contenidos no puede lograse a expensas de una renuncia a un conocimiento estructurado y profundo.

e) Priorizar los aprendizajes básicos.

- Establecimiento de un plan especial para la mejora en Matemáticas.

- Establecimiento de un plan especial para la mejora en Lectura.

- Definición de cuatro pilares formativos básicos: lectura y escritura fluidas; matemáticas, estadística y lógica; historia y geografía; y ciencias.

- Definición de dos áreas adicionales: artes en sus formas diversas; y literatura y lenguas.

- Orientación del currículo de un modo flexible y moderno.

f) Alinear los recursos con la reforma del currículo.

- Movilización de los recursos de conocimiento experto basados en los avances científicos al respecto.

- Establecimiento de una nueva normativa sobre libros de texto para convertirlos en uno de los pilares esenciales en los que apoyar la reforma curricular.

g) Reforzar la autonomía de los centros.

- Organización autónoma de las horas de dotación de profesorado asignado por la administración.

- Organización de las asignaturas de acuerdo con una lógica de curso o de ciclo.

- Asignación de sus recursos en la implementación de medidas para promover el éxito escolar.

- Ante la escasez de recursos durante la intervención de su economía, desarrollo de incentivos de orden moral, tales como premios o reconocimientos públicos.

- Reconocimiento de los centros que mejoren los resultados de sus alumnos, mediante la ampliación del número de horas de dotación de profesorado disponible para el centro.

h) Fortalecer la profesión docente.

- Introducción de un examen de entrada para los candidatos a profesor.

- Mejora de la formación inicial del profesorado, mediante la regulación del acceso y la reforma de los programas con el refuerzo de los conocimientos de las disciplinas básicas. 
Establecimiento de un máster de dos años con un amplio periodo de inducción.

i) Adoptar un enfoque propio de un gradualismo reformador que, bajo gobiernos de signo diferente, otorga una continuidad constructiva a las reformas.

La OCDE (2018), a propósito de su análisis experto sobre la reforma que ha puesto en marcha Portugal para orientar sus currículos, de conformidad con el enfoque por competencias (Proyecto de Autonomía y Flexibilidad Curricular), efectúa la siguiente valoración general de la educación portuguesa:

Portugal ha adoptado un enfoque estratégico sólido para la reforma educativa. El país comenzó el proceso concibiendo los resultados que el sistema educativo debería buscar para sus alumnos, basándose en evidencias sobre las condiciones propias del contexto del siglo XXI. Formuló estos objetivos en un plan estratégico coherente, descrito con detalle en el documento de referencia, "Perfil de los estudiantes al finalizar la escolarización obligatoria". Portugal ha logrado un acuerdo general sobre sus planes de reforma a través de consultas, debates y comunicaciones cuidadosas que han sido bien gestionadas y exitosas. Al buscar el asesoramiento de expertos, las aportaciones de los actores y la comunicación y el debate abiertos, el país ha invertido en la continuidad del plan de reforma por parte de los futuros gobiernos. El país parece estar abierto a la retroalimentación y al aprendizaje de las lecciones derivadas de las evidencias que han surgido sobre los éxitos y las debilidades del 'Proyecto de Autonomía y Flexibilidad Curricular (OECD, 2018, p. 7).

Más allá de las decisiones puntuales -aunque no por ello menos relevantes- adoptadas por Portugal en materia de políticas educativas, hay un rasgo muy general que cabe inferir de lo anterior y que explicaría todo lo demás. Y es, pura y simplemente, la calidad de su gobernanza en educación. Gobernanza que contiene buena parte de los rasgos propios de un gobierno inteligente de los sistemas educativos (López Rupérez et al., 2017).

\subsubsection{Algunas características comparadas de las reformas educativas españolas}

Con el propósito de facilitar la comparación, cabe tomar en consideración los 9 criterios descritos más arriba y que sintetizan los principios inspiradores de las reformas portuguesas desarrolladas a lo largo del presente siglo. No obstante, resulta difícil encontrar puntos de coincidencia. Así, por ejemplo, España no ha hecho de los resultados y de su mejora (a) una prioridad explicita de su sistema educativo, ni ha planteado en sus reformas-traducidas en las leyes orgánicas de 2006 (LOE) y 2013 (LOMCE)- esa ambición por el conocimiento $(d)$ que alcance a todos, sea cual fuere su nivel socioeconómi- 
co y cultural (c); ni tampoco ha hecho suya la recomendación de 2015 del Consejo Escolar del Estado de extender la "enseñanza básica" (obligatoria y gratuita) hasta los 18 años (b) (CEE, 2015; López Rupérez et al., 2015).

Si se profundiza en las reformas propiamente curriculares (e y f), España se ha alejado de esas claves del éxito portugués que ha diagnosticado la OCDE (2018). Así, y como hemos destacado y justificado en otro lugar, al analizar la reforma curricular de la LOMCE y su enfoque por competencias (López Rupérez, 2020):

La contrastación del caso de España con las mejores prácticas internacionales ha permitido identificar cuatro tipos de errores:

a) La apuesta por una opción de máximos y no gradualista del Marco Europeo de Referencia (todas las competencias, todas las materias, todas las etapas y al mismo tiempo), reflejo probablemente de una ambición política por alinearse con las orientaciones de la Unión Europea que ha resultado ser a la postre excesiva, simple y poco crítica.

b) Una confianza excesiva, de inspiración burocrática, en el poder transformador de las normas, al considerar la publicación de los currículos en el Boletín Oficial del Estado como la culminación de la labor del Gobierno.

c) La debilidad de un planteamiento estratégico que no contempló una aproximación sistémica o integrada de diferentes políticas facilitadoras del éxito en la implementación de la reforma curricular; ni se articuló ningún plan estratégico, siquiera fuere parcial, que las incorporara con realismo; ni se implicó suficientemente al profesorado, ni a los directores en el proceso.

d) Un insuficiente liderazgo institucional, escasamente basado en las personas y en el conocimiento e incapaz de pilotar -desde la auctoritas y no sólo desde la potestas- los procesos de reforma del sistema en su conjunto, justamente en un difícil contexto político con riesgos permanentes de conflicto entre administraciones gobernadas por partidos de distinto signo, que suelen emplear la confrontación con el Gobierno como una estrategia de desgaste político del adversario." (López Rupérez, 2020, p.145)

Algunos tímidos avances normativos se pueden apreciar, de la mano de la LOMCE, en materia de autonomía de los centros (g), así como en los procesos de selección de los directores escolares en los centros públicos. Pero ningún progreso normativo se ha producido en materia de fortalecimiento de la profesión docente (h), más allá de los debates -prolongados en el tiempo, a lo largo de la última década- sobre el Ilamado "MIR educativo" (López Rupérez, 2014; 2015). 
En materia de calidad de la gobernanza del sistema educativo español, una evaluación de carácter exploratorio apoyada en un estudio Delphi (López Rupérez et al., 2017; 2020) -sobre un panel de 21 expertos y tres rondas sucesivas de consultas- ha arrojado los siguientes resultados:

Para ninguna de las seis categorías consideradas en el modelo la puntuación total alcanza un valor igual o superior a 2,0 puntos -que corresponde a la calificación de mediano- lo que revela, a juicio de los expertos, una notable y consistente mediocridad de la gobernanza de nuestro sistema educativo. La dimensión n. ${ }^{\circ}$ 4, «Gobernanza basada en el conocimiento, la evidencia empírica y la investigación», por su baja calidad, sobresale, de un modo consistente, sobre todas las demás. Su puntuación igual a 1,5 la sitúa en un nivel claramente mediocre, es decir, entre escaso y mediano". (López Rupérez et al., 2017, p. 102).

\section{Conclusiones}

Del análisis comparado entre los sistemas educativos de España y Portugal, efectuado a partir de un conjunto amplio de indicadores mayoritariamente cuantitativos, se derivan, en síntesis, las siguientes conclusiones empíricas:

a) En relación con el contexto, España se sitúa consistentemente por encima de Portugal en factores tales como nivel de riqueza y estatus socioeconómico y cultural.

b) En lo que concierne a los inputs, el gasto acumulado por alumno ente los 6 y los 15 años Portugal supera a España en un 11\% aproximadamente, aunque la percepción de escasez de recursos -tanto educativos como humanos- por parte de los directores de los centros portugueses, es superior; probablemente como consecuencia de carencias procedentes del pasado y aún no cubiertas.

c) En materia de outputs, en todos los indicadores de resultados educativos considerados - puntuaciones en PISA, tasa de la población adulta joven que ha completado al menos la educación secundaria superior, y brecha formativa de la población adulta joven- Portugal ha conseguido, a lo largo de la última década, superar a España.

d) En lo relativo a los outcomes analizados -abandono educativo temprano, tasa de NiNis y riesgo de pobreza y exclusión socialPortugal se sitúa en posiciones sistemáticamente más favorables 
que las de España y, en algún caso, que las de la media de los países de la UE.

e) Finalmente, en lo que respecta a las políticas que transforman los inputs en outputs, la posición de Portugal está claramente más adelantada que la de España, según se infiere de un análisis comparado, de naturaleza cualitativa, basado tanto en descripciones de académicos portugueses, como en evaluaciones de la OCDE.

A la vista de los análisis más arriba descritos, cabe establecer como conclusión principal que España debería emprender reformas profundas y continuadas de su sistema educativo inspiradas en el consenso internacional emergente. Ello requeriría:

a) Un planteamiento político y estratégico adecuado que garantice un programa consensuado de reformas, extendido sobre una década.

b) Un apoyo claro de dicho programa en el conocimiento, la evidencia empírica y la investigación

c) Una priorización de aquellas políticas que más impacto tienen sobre los resultados de los alumnos.

d) Un enfoque integrado, entendido como aquél que toma en consideración esos factores críticos junto con sus interrelaciones.

e) Una aportación de recursos adicionales al sistema con criterios de eficacia, eficiencia y equidad.

f) Una implicación efectiva de los actores principales a fin de contribuir al éxito de las reformas.

Por su carácter general, estas recomendaciones son de aplicación en aquellos países de Iberoamérica que hayan de emprender procesos de reforma de sus sistemas educativos.

Procede en este punto final traer a colación la conclusión de Ben Levin (2010) -basada no sólo en la reflexión académica, sino también en su experiencia en la acción política- que resumía las lecciones de los últimos 50 años en materia de reformas educativas en los siguientes términos: "Si hay una cosa que hemos aprendido sobre política educativa es que ordenar a la gente que lo haga mejor no podrá tener éxito sin comprometer sus corazones y sus mentes" (Levin, 2010, p.742). 


\section{Referencias}

CEE (2015). Informe 2015 sobre el estado del sistema educativo. Consejo Escolar del Estado. Ministerio de Educación Cultura y Deporte. Madrid. Disponible en https://bit. ly/347Gvwr

CERI-OECD (1992). The OECD International Education Indicators. A Framework for analyses. Paris: OECD Publishing.

CERI-OECD (1994). Évaluer l'enseignement. De l'utilité des indicateurs internationaux. Paris: Éditions OECD.

CERI-OECD (2007). Comprendre l'impact social de l'éducation. Paris: Éditions OECD.

Crato, N. (2020). Curriculum and Educational Reforms in Portugal: An Analysis on Why and How Students' Knowledge and Skills Improved. Audacious Education Purposes. How Governments Transform the Goals of Education Systems. Reimers, F.M. (Ed). Chum, Switzerland: Springer. Disponible en https://bit.ly/36frag5

European Commission (2019). Education and Training Monitor 2019. Dsiponible en https:// bit.ly/3jgr9Ms

Eurostat (2020). People at risk of poverty or social exclusion by sex. Disponible en https://bit. Iy/3mXOqVL

Eurydice (2015). Compulsory Education in Europe, 2014/15. Disponible en https://bit.ly/2S87geH

Levin, B. (2010). Governments and education reform: some lessons from the last 50 years. Journal of Educational Policy, 25:6, 739-747. https://doi.org/10.1080/0268093 $\underline{9.2010 .523793}$

López Rupérez, F. (1994). La gestión de calidad en educación. Madrid: La Muralla.

López Rupérez, F. (2014). Fortalecer la profesión docente. Un desafío crucial. Madrid: Narcea Ediciones

López Rupérez, F. (2015). "MIR Educativo" y profesión docente. Un enfoque integrado. Revista Española de Pedagogía, LXXIII(261), 283-299. Disponible en https://bit.ly/30gOTbP

López Rupérez, F. (2020). El currículo y la educación en el siglo XXI. La preparación del futuro y el enfoque por competencias. Madrid: Narcea Ediciones.

López Rupérez, F., García García, I. y Sanz Labrador, I. (2015). La extensión de la enseñanza básica hasta los 18 años. Beneficios y costes. Madrid: Fundación para los análisis y los estudios sociales. Disponible en https://bit.ly/34bYfXPf

López Rupérez, F., García García, I. y Expósito Casas, E. (2017). La calidad de la gobernanza del sistema educativos español. Un estudio empírico. Madrid: Universidad Camilo José Cela. Disponible en https://bit.ly/30ihP3I

López Rupérez, F., García García, I. y Expósito Casas, E. (2019). Liderazgo de la dirección y feedback formativo. Dos pilares básicos de la gobernanza escolar. Madrid: Universidad Camilo José Cela. Disponible en https://bit.Iy/33a6KTG

López Rupérez, F., García García, I. y Expósito Casas, E. (2020). Un marco analítico para la evaluación de la calidad de la gobernanza de los sistemas educativos. Revista Iberoamericana de Educación, 83(1), 53-76. https://orcid.org/0000-0003-2613$\underline{9652}$ 
López Rupérez, F. y García García, I. (2020). A vueltas con la equidad en educación. Una aproximación empírica en la perspectiva de las consecuencias. Madrid: Universidad Camilo José Cela.

OECD (2012). Education at a Glance. OECD Indicators. Paris: OECD Publishing.

OCDE (2016). Résultats du PISA 2015 (Volume I): L'excellence et l'équité dans l'éducation. PISA. Paris: Éditions OCDE. https://doi.org/10.1787/9789264267534-fr

OECD (2018). Curriculum Flexibility and Autonomy in Portugal. An OECD review. Disponible en https://bit.ly/33cX2jr

OECD (2019). PISA 2018 Results (Volume I): What Students Know and Can Do. PISA. Paris: OECD Publishing. https://doi.org/10.1787/5f07c754-en

Parlamento Europeo (2011). Abordar el abandono escolar prematuro. Diario oficial de la Unión Europea. Resolución del Parlamento Europeo, de 1 de diciembre de 2011. Disponible en https://bit.ly/3mXRjWB

Reimers, F. \& Schleicher, A. (2020). A framework to guide an education response to the COVID-19 Pandemic of 2020. Paris : OECD. Disponible en https://bit.ly/3kT9efp

Schleicher, A. (2018). Primera Clase. Cómo construir una escuela de calidad para el siglo XXI. Madrid: Fundación Santillana.

Shwab, K. (2016). Cuatro principios de liderazgo de la cuarta revolución industrial. Foro Económico Mundial. Disponible en https://bit.ly/33anQ3S

Xia, N. \& S.N. Kirby (2009). Retaining Students in Grade: A Literature Review of the Effects of Retention on Students' Academic and Nonacademic Outcomes. RAND Technical report. Disponible en https://bit.ly/3cleShi 